\title{
HIGHLIGHTS
}

BILIARY TRACT

\section{A new duct-to-duct reconstruction technique reduces biliary complications in LDLT}

A new method for biliary reconstruction in living donor liver transplantation (LDLT) has resulted in considerably improved outcomes in comparison with the conventional technique. Kim et al. developed the new method-tailored telescopic reconstruction (TTR) - because previous advances in this field had not reduced the substantial morbidity and mortality associated with biliary

Preparation of the funnel-shaped top in the recipient bile duct. Image courtesy of K.-W. Lee.

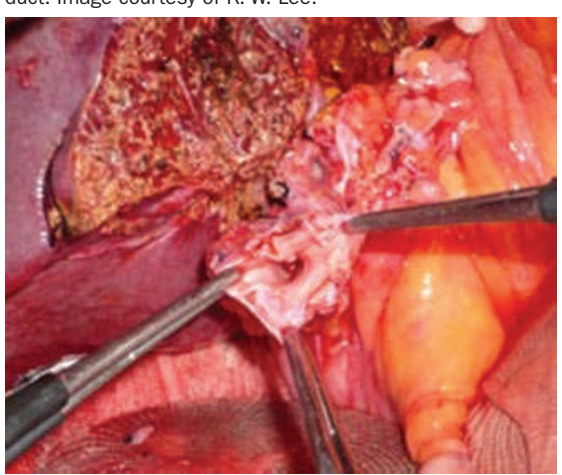

reconstruction in LDLT. "It is hard to develop novel techniques to improve the biliary outcome in duct-to-duct anastomosis," explains Kwang-Woong Lee, the study's lead investigator. "Using this new TTR technique, however, we can significantly reduce biliary complications."

TTR involves bisecting the hilar plate lengthwise through the left or right hepatic duct to make a funnel-shaped top. Next, the donor hepatic duct is telescoped to match the size of the recipient bile duct and duct-to-duct reconstruction is performed with the inner tissue of the recipient bile duct being used for anastomosis. The researchers tested the TTR technique on 22 patients undergoing LDLT and compared the outcomes at a mean of 19.5 months with those from conventional duct-toduct reconstructions in 23 individuals. Overall, there were significantly less biliary complications in patients who had undergone TTR as opposed to the conventional technique $(9.1 \%$ versus
$43.5 \%$, respectively; $P<0.05)$. Lee's group also found the rates of biliary leakage and strictures to be $0 \%$ and $9.1 \%$, respectively, for TTR in comparison with $26.1 \%$ and $34.8 \%$ for the conventional technique.

The investigators attribute much of the success of TTR to the fact that it was associated with no biliary leakage; a direct consequence of utilizing the inner tissue of the recipient bile duct for anastomosis. However, they stress that long-term outcomes were not addressed in this study and will need to be closely monitored before the true potential of TTR is established. "TTR alone is not enough to eliminate the biliary complications because multiple factors can affect the outcome," notes Lee.

Rowan Higgs

Original article Kim, S. H. et al. Tailored telescopic reconstruction of the bile duct in living donor liver transplantation. Liver Transpl. 16, 1069-1074 (2010) 